\title{
Reliability and validity of pediatric triage tools evaluated in Low resource settings: a systematic review
}

\author{
Bhakti Hansoti ${ }^{*}$ D, Alexander Jenson ${ }^{1}$, Devin Keefe', Sarah Stewart De Ramirez ${ }^{1}$, Trisha Anest ${ }^{1}$, Michelle Twomey ${ }^{2}$, \\ Katie Lobner ${ }^{3}$, Gabor Kelen ${ }^{1}$ and Lee Wallis ${ }^{2}$
}

\begin{abstract}
Background: Despite the high burden of pediatric mortality from preventable conditions in low and middle income countries and the existence of multiple tools to prioritize critically ill children in low-resource settings, no analysis exists of the reliability and validity of these tools in identifying critically ill children in these scenarios.

Methods: The authors performed a systematic search of the peer-reviewed literature published, for studies pertaining to for triage and IMCl in low and middle-income countries in English language, from January 01, 2000 to October 22, 2013. An updated literature search was performed on on July 1, 2015. The databases searched included the Cochrane Library, EMBASE, Medline, PubMed and Web of Science. Only studies that presented data on the reliability and validity evaluations of triage tool were included in this review. Two independent reviewers utilized a data abstraction tool to collect data on demographics, triage tool components and the reliability and validity data and summary findings for each triage tool assessed.
\end{abstract}

Results: Of the 4,717 studies searched, seven studies evaluating triage tools and 10 studies evaluating IMCI were included. There were wide varieties in method for assessing reliability and validity, with different settings, outcome metrics and statistical methods.

Conclusions: Studies evaluating triage tools for pediatric patients in low and middle income countries are scarce. Furthermore the methodology utilized in the conduct of these studies varies greatly and does not allow for the comparison of tools across study sites.

\section{Background}

The global burden of pediatric mortality in low resource settings remains high; 6.3 million children under five years old die worldwide each year. Although under-five mortality has declined from 90 to 43 deaths per 1,000 live births since 1990, improvements have fallen short of Millennium Development Goal (MDG) 4 which called for a two-thirds reduction in mortality worldwide by 2015 [1]. A majority of childhood deaths are attributable to easily treatable, time sensitive illness [2]. It is estimated that as much as $60 \%$ of mortality in this population may be reduced by improving access to care [3]. Providing timely

\footnotetext{
* Correspondence: Bhansot1@jhmi.edu

${ }^{1}$ Department of Emergency Medicine, Johns Hopkins School of Medicine,

1830 Monument St Suite 6-100, Baltimore, MD 21287, USA

Full list of author information is available at the end of the article
}

access to specialized emergency care has been shown in numerous settings to confer a mortality benefit [4].

Triage is the prioritization of patients, usually to identify the sickest for earliest intervention; it typically consists of a complex decision-making process including clinical discriminators, physiological parameters, or both [5]. Triage has the ability to substantially decrease pediatric mortality and morbidity by providing timely care for critically ill patients [6]. Several validated scales exist; however, much of the triage data is derived from high-income countries [7].

In recent years, there has been a push to develop triage scales specifically tailored to low resource environments in Low and Middle Income Countries (LMICs) [8]; examples include tools such as the Pediatric South African Triage Scale (PSATS) [9] and the WHO Emergency Triage and Treatment Tool (ETAT) [10] among others. In the clinic setting healthcare workers utilize the WHO developed 
and implemented Integrated Management of Childhood Illnesses (IMCI) to identify pediatric patients with time sensitive illness requiring urgent treatment and/ or referral $[2,3]$.

Although not a traditional triage tool, the IMCI is a well studied and widely implemented in both out patient clinic and hospital settings. Therefore, the authors' felt that studies evaluating the IMCI in LMICs warranted specific consideration. It is imperative that healthcare providers and policy makers understand the evidence and generalizability of the evaluation studies of these tools, among others, prior to implementation. This systematic review aims to investigate the scientific evidence underlying the use of acute care triage scales and IMCI for pediatric patients in LMICs.

\section{Methods}

\section{Search strategy}

The authors performed a systematic search of the peerreviewed literature published, in English language, from January 01, 2000 to October 22, 2013, with an updated literature search on July 1, 2015. The databases searched included the Cochrane Library, EMBASE, Medline, PubMed and Web of Science.

Two separate searches were conducted. Both searches included search filters for LMICs (Additional file 1: Appendix A). The first search included the Medical Subject Heading (MESH) term "triage", and a separate search was conducted for the Integrated Management of Childhood Illness (IMCI). Income status of the country was defined by World Bank criteria [11]. All applicable controlled vocabularies and keyword terms were searched in all databases. The search was run without any restrictions and two authors screened each result. All studies pertaining to the evaluation of triage tools for pediatric patients $(<18$ years of age), in an acute care setting (i.e., where undifferentiated patients present for care), were included in the review. We included studies conducted in both hospitals and clinic settings.

Studies were excluded if the described tool was not designed to affect patient treatment or destination (i.e. a trauma score), if the tool was disease specific, or if the article was not available in English. Various study designs were included such as randomized control trials, observational studies and descriptive studies, however case reports or case series (defined as $n<5$ ) were, excluded (Additional file 2: Appendix B).

Each study included in the review underwent data abstraction using a data abstraction tool (Additional file 3: Appendix C) by two independent reviewers. We collected data on four elements, (1) the demographics of the study locale, (2) the triage tool components, (3) the reliability data and (4) the validity data and summary findings for each triage tool assessed. Evaluations of the studies, including the risk of bias or an evaluation of quality of the individual study methodology, was not a component of the data abstraction tool for this review.

\section{Outcome variables}

Reliability was defined as the assessment of triage tools against other evaluations, either by another health care professional (inter-rater), or a triage tool expert designer/ study author (expert opinion). Validity was defined as evaluation of outcomes for triaged patients (admission, ICU admission, referral, death etc.) by triage category. Multiple studies included assessments of "over" and "under" triage, but given the heterogeneity of these definitions $[12,13]$ and different methods of computing the result across studies $[13,14]$, these analyses were not included in the review.

\section{Results}

The initial search strategy returned 4,717 results, with 2,742 unique articles (Fig. 1). Each title was then assessed for inclusion based on the specific criteria above and was analyzed by two independent reviewers; a third senior author evaluated articles with discordant results.

After the initial title review, 411 abstracts were identified, including triage of both adult and pediatric patients. A total of seven studies evaluating triage tools in pediatric patients and 10 studies evaluating IMCI are included in the review.

\section{Study locale}

Seven studies assessed a total of five triage tools in pediatric acute care settings in LMICs. Only one of the tools (ETAT) was evaluated in a low income country (Malawi) [15]. All of the remaining tools were evaluated in middle-income countries, with four tools (PEWS, PSATS, ETAT, ESI) $[10,16-18]$ exclusively evaluated in upper-middle income countries. Five studies were conducted in tertiary care centers, and one study was conducted in a district hospital setting [10]. Only one study was multi-center, with Twomey et al [18] evaluating PSATS in 5 hospitals and 1 community health center.

IMCI evaluations were exclusively conducted in lower middle-income countries (India, Bolivia, and Vietnam) [19-28]. One of the ten studies was conducted in exclusively outpatient settings [28], five were conducted in hospital EDs [19-22, 26], and four were conducted in both settings as a multi-center evaluation.

\section{Tool components}

The tools included for pediatric triage had varying components, and as a result varying levels of complexity (Table 1). The ETAT guidelines involve triage of patients according to emergency and priority signs using an $\mathrm{ABCD}$ concept (Airway, Breathing, Circulation/Coma/Convulsion, Dehydration), and rely on clinical discriminators rather than 


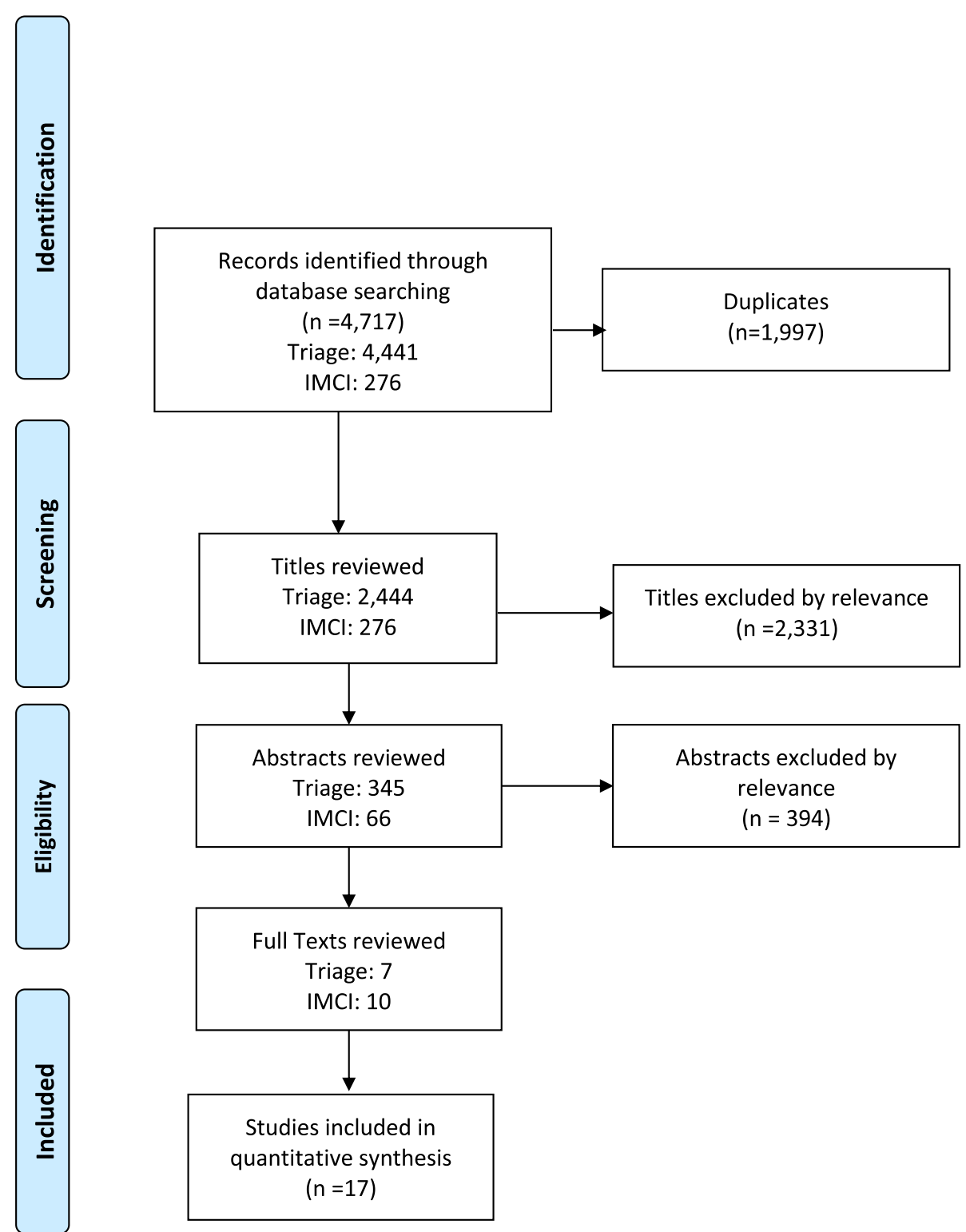

Fig. 1 PRISMA flow diagram of search methodology

Table 1 Overview of study local, study type and triage tool components included in the systematic review

\begin{tabular}{|c|c|c|c|c|c|c|c|c|c|c|c|c|c|}
\hline \multirow[t]{2}{*}{ Tool } & \multirow[t]{2}{*}{ Studies } & \multirow[t]{2}{*}{ Evaluations } & \multicolumn{2}{|c|}{ Country Setting } & \multicolumn{3}{|c|}{ Study Locale } & \multicolumn{2}{|c|}{ Study Type } & \multicolumn{4}{|c|}{ Tool Components } \\
\hline & & & $\begin{array}{l}\text { Middle } \\
\text { Income }\end{array}$ & $\begin{array}{l}\text { Low } \\
\text { Income }\end{array}$ & $\begin{array}{l}\text { Tertiary } \\
\text { Hospital }\end{array}$ & $\begin{array}{l}\text { District } \\
\text { Hospital }\end{array}$ & $\begin{array}{l}\text { Outpatient } \\
\text { Clinic }\end{array}$ & Reliability & Validity & $\begin{array}{l}\text { Vital } \\
\text { Signs }\end{array}$ & $\begin{array}{l}\text { Clinical } \\
\text { Discriminators }\end{array}$ & $\begin{array}{l}\text { Presenting } \\
\text { Complaint }\end{array}$ & $\begin{array}{l}\text { Resources } \\
\text { Required }\end{array}$ \\
\hline pSATS $^{a}$ & 2 & 3 & $x$ & & $x$ & & $x$ & & $x$ & $x$ & $x$ & $x$ & \\
\hline $\mathrm{ETAT}^{\mathrm{b}}$ & 2 & 2 & $x$ & $x$ & $x$ & $x$ & $x$ & $x$ & $x$ & & $x$ & & \\
\hline ESI & 1 & 2 & $x$ & & $x$ & & & $x$ & $x$ & $x$ & $x$ & $x$ & $x$ \\
\hline PEWS & 1 & 4 & $x$ & & $x$ & & & $x$ & $x$ & & $x$ & & \\
\hline TOPRS & 1 & 1 & $x$ & & $x$ & & & & $x$ & $x$ & & & \\
\hline $\mathrm{IMCl}$ & 10 & 12 & $x$ & & $x$ & $x$ & $x$ & $x$ & $x$ & $x$ & $x$ & $x$ & \\
\hline
\end{tabular}

ancludes both pSATS and PATS, a modification of SATS with minor changes

bincludes both ETAT and abbreviated ETAT 
physiologic parameters to stratify sick children [10]. Conversely, the PEWS (Pediatric Early Warning Score) and TOPRS (Temperature, O2 saturation, Pulse, Respiratory rate, Sensorium/Seizures) relies solely on physiologic parameters (vital signs) to predict hospitalization [16, 29]. Some tools combined both physiologic parameters and clinical discriminators such as the Pediatric South African Triage Scale (pSATS) which incorporates the ETAT ABCD emergency signs as well as Triage Early Warning Score (TEWS) physiologic parameters to stratify patients. Lastly, the Emergency Severity Index (ESI) adds further complexity by asking the provider the number of resources that will be required.

IMCI utilizes a syndrome-based approach to target the care of children, and thus uses physiologic and clinical discriminators in evaluating triage category. This is because, although the IMCI strategy does have a prioritization component (so that critically ill children may be transferred to a higher level of care), its stated purpose is to promote curative care in the outpatient setting, and includes algorithms for healthcare workers to direct care for common complaints in a pediatric outpatient settings.

\section{Pediatric triage tools \\ Reliability}

Three studies measured the reliability of three different triage tools (Table 2) $[7,16,17]$. Roberston et al. evaluated ETAT in Malawi and found that nurses have an agreement of $93.8 \%$, assigning the correct triage level, when compared against physician "assessors" observing triage [7]. The ESI evaluation by Jafari-Rouhi et al. utilized blinded re-rating by pediatric emergency medicine physicians to assess nursing performance with an overall kappa of 0.82 [17]. Both studies included real patients with real-time triage evaluations in a clinical setting, and all studies were appropriately blinded to the other provider's assessment. The highest percentage agreement was achieved in the level 1 /priority 1 patient group.

\section{Validity}

All seven triage studies included some measurement of validity $[4,7,10,16-18]$. The studies included, tools evaluated, and outcome measures utilized are shown in Table 3.
Two studies analyzed the likelihood of admission by ETAT triage level assigned. Ninety percent of priority 1 (P1 or critically ill) patients were admitted versus $32 \%$ of P2 and 4.5\% of P3 in one study [7]. Another group published similar percentages with a significant increase in the relative risk of admission in children triaged to level 1 or 2 compared to 3 (RR 2.6; $95 \%$ CI 2.2-3.1 in one sample and RR 3.2; 95\% CI 2.5-4.1 in another) [10]. Using the pSATS tool, Twomey et al. reported an increase in hospital admission with increasing level of urgency from $4.7 \%$ in the lowest triage level to $72.8 \%$ of those triaged to level 1 [18]. The area under the ROC curve for predicting overall admission using PEWS was 0.73 . PEWS was $100 \%$ sensitive and $90.5 \%$ specific for predicting ICU admission [16]. In a large study conducted by Mullan et al. analyzed both adult and pediatric ( $<13$ years old) patients using PATS, a modified form of SATS. They demonstrated an increase in hospital admission (20.6-86.7\%) and mortality (0-1.4\%) when children were assigned a higher acuity score [30, 31].

Using the ESI v.4 in Iran, 100\% of patients assigned level 1 by pediatric EM physicians were either admitted to the ICU or died while $0 \%$ were admitted to the ward or discharged [17]. Of level 2 patients, $1.2 \%$ were admitted to the ICU or died, $29.8 \%$ were admitted to the ward, and $69 \%$ were discharged. Zero level 3 through 5 patients went to the ICU or died [17]. The TOPRS score was found to have a predictive ability of $81.7 \%$ for admission on receiver operating curve analysis with a progressive increase in mortality by increasing score.

\section{IMCI/IMNCI}

\section{Reliability}

Six of the included studies published Kappa or percent agreement data to quantify the concordance of nurse or health worker IMCI determination with physician diagnosis (Table 4) [19-23, 28]. There was a large variation in the overall Kappa values, from 0.16 to 0.59 [19, 26]. Bhattacharya et al., 2011, reported a syndrome specific kappa and found the highest agreement in the diagnosis of jaundice (0.73) and the lowest agreement in the diagnosis of dehydration (0.19) [21].

Table 2 Included reliability studies of pediatric triage tools

\begin{tabular}{|c|c|c|c|c|}
\hline Triage Tool & $\begin{array}{l}\text { Author[ref] Year Country } \\
\text { (Income level) }\end{array}$ & Comparison Groups & $\begin{array}{l}\text { Volume Patient and Setting } \\
\text { Characteristics (Age restrictions) }\end{array}$ & $\begin{array}{l}\text { Results (kappa, percent } \\
\text { agreement) by triage level }\end{array}$ \\
\hline ETAT & $\begin{array}{l}\text { Robertson etal. [7] } 2001 \\
\text { Malawi (Low Income) }\end{array}$ & $\begin{array}{l}\text { 1. Clinic nurse } \\
\text { 2. ED physician }\end{array}$ & $\begin{array}{l}n=2281 \text { Under } 5 \text { outpatient clinic } \\
(85 \%<5 y / 0)\end{array}$ & $\begin{array}{l}\text { Overall: } 94.8 \% \text { P1: } 95.7 \% \\
\text { P2: } 88.0 \% \text { P3: } 96.1 \%\end{array}$ \\
\hline ESI & $\begin{array}{l}\text { Jafari-Rouhi etal. [17] } 2013 \\
\text { Iran (Upper Middle Income) }\end{array}$ & $\begin{array}{l}\text { 1. Triage nurse to Ped } \\
\text { 2. ED physician }\end{array}$ & $\begin{array}{l}n=1104 \text { Emergency department at } \\
\text { national teaching hospital }(100 \%<18 \mathrm{y} / 0)\end{array}$ & $\begin{array}{l}\text { Kappa } 0.82 \text { Overall } 87.3 \% \\
\text { Level 1: } 100 \% \text { Level 2: } 93.1 \% \\
\text { Level 3: } 83.4 \% \text { Level 4: } 86.1 \% \\
\text { Level 5: } 84.1 \%\end{array}$ \\
\hline PEWS & $\begin{array}{l}\text { Chaiyakulsil etal. [16] } 2015 \\
\text { Thailand (Upper Middle income) }\end{array}$ & $\begin{array}{l}\text { 1. Triage nurse } \\
\text { 2. Triage nurse }\end{array}$ & $\begin{array}{l}n=1136 \\
E D \text { at large tertiary care hospital }\end{array}$ & Kappa: 0.75 \\
\hline
\end{tabular}


Table 3 Included validation studies of pediatric triage tools

\begin{tabular}{|c|c|c|c|c|c|}
\hline Outcome & Scale & $\begin{array}{l}\text { Author [ref] Year Country } \\
\text { (Income) }\end{array}$ & $\begin{array}{l}\text { Site Volume Patient and Setting } \\
\text { Characteristics (Age restrictions) }\end{array}$ & $\begin{array}{l}\text { Results (per triage } \\
\text { level) }[p \text { value }]\end{array}$ & Remarks \\
\hline \multirow[t]{3}{*}{ Mortality } & ESI & $\begin{array}{l}\text { Jafari-Rouhi [17] } 2013 \\
\text { Iran (Upper Middle } \\
\text { Income) }\end{array}$ & $\begin{array}{l}n=1104 \mathrm{ED} \text { at national teaching } \\
\text { hospital. }(100 \%<18 \mathrm{y} / \mathrm{o})\end{array}$ & $\begin{array}{l}\text { Overall: } 0.9 \% \text { 1: } \\
\text { 100\% 2: } 1.2 \% \\
\text { 3:0\% 4:0\% 5: } 0 \%\end{array}$ & $\begin{array}{l}\text { Outcome was ICU admission or death. } \\
\text { ESI performed at patient presentation, } \\
\text { not admission. }\end{array}$ \\
\hline & TOPRS & $\begin{array}{l}\text { Bains } 2012 \text { [15] India } \\
\text { (Lower-middle Income) }\end{array}$ & $\begin{array}{l}n=777 \text { Teaching hospital with } \\
\text { general ED. }\end{array}$ & $\begin{array}{l}\text { Overall: } 16.3 \% \\
\text { TORPS 6: } 100 \% \\
\text { TORPS 5: } 80.0 \% \\
\text { TORPS 4: } 66.7 \% \\
\text { TORPS 3: } 60.0 \% \\
\text { TORPS 2: } 38.2 \% \\
\text { TORPS 1: } 12.5 \% \\
\text { TOPRS 0: } 4.4 \%\end{array}$ & $\begin{array}{l}\text { All patients were admitted to ED. ROC curve } \\
\text { maximal discrimination at } 2.5 \text { (sensitivity } \\
79.6 \% \text {, specificity } 74.3 \% \text { ) }\end{array}$ \\
\hline & PATS & $\begin{array}{l}\text { Mullan } 2014 \text { [ ] Botswana } \\
\text { (Upper-middle Income) }\end{array}$ & $\begin{array}{l}n=4466 \\
E D \text { at terteriary referral hospital } \\
(100 \%<13 \text { y/o })\end{array}$ & $\begin{array}{l}\text { Overall: } 0.16 \% \\
\text { Red: } 1.4 \% \\
\text { Orange: } 0.05 \% \\
\text { Yellow: } 0 \% \\
\text { Green: } 0 \%\end{array}$ & $\begin{array}{l}\text { Outcome was ICU admission or death. PATS } \\
\text { performed at ED presentation. Large study of } \\
\text { both adult and pediatric patients with } \\
\text { separate analyses. }\end{array}$ \\
\hline \multirow[t]{6}{*}{ Admission } & ESI & $\begin{array}{l}\text { Jafari-Rouhi } 2013 \text { [17] } \\
\text { Iran (Upper-middle } \\
\text { Income) }\end{array}$ & $\begin{array}{l}n=1104 \mathrm{ED} \text { at national teaching } \\
\text { hospital. }(100 \%<18 \mathrm{y} / \mathrm{o})\end{array}$ & $\begin{array}{l}\text { Overall: } 9.4 \% \\
\text { 1: } 0 \% \text { 2: } 29.8 \% \\
\text { 3: } 1.8 \% 4: 2.0 \% \\
\text { 5: } 0 \%\end{array}$ & $\begin{array}{l}\text { Outcome was ED admission or ward } \\
\text { admission (does not include ICU admission). } \\
\text { Spearman correlation coeficient } 0.407 \text {. }\end{array}$ \\
\hline & $\begin{array}{l}\text { Adapted } \\
\text { ETAT }\end{array}$ & $\begin{array}{l}\text { Buys } 2013 \text { [10] South } \\
\text { Africa (Upper-middle } \\
\text { Income) }\end{array}$ & $\begin{array}{l}n=407 \text { District hospital with } \\
\text { general ED. }(100 \%<16 y / 0)\end{array}$ & $\begin{array}{l}\text { Overall: } 24.8 \% \text { P1: } \\
\text { 91.7\% P2: } 36.9 \% \\
\text { P3: } 10.1 \%\end{array}$ & $\begin{array}{l}\text { Second of } 2 \text { cohorts (2009), first (2007) } \\
\text { immediately following training. }\end{array}$ \\
\hline & pSATS & $\begin{array}{l}\text { Twomey } 2013 \text { [18] South } \\
\text { Africa (Upper-middle } \\
\text { Income) }\end{array}$ & $\begin{array}{l}n=20146 \text { ED centers with } \\
\text { varying size/populations. }(100 \% \\
<13 \text { y/o) }\end{array}$ & $\begin{array}{l}\text { Overall: } 21.5 \% \text { 1: } \\
72.8 \% \text { 2: } 29.0 \% 3: \\
27.9 \% \text { 4: } 4.7 \%\end{array}$ & $\begin{array}{l}\text { Sensitivity } 91.0 \% \text {, Specificity } 54.5 \% \text {. } \\
\text { Compared to simply TEWS or clinical } \\
\text { discriminator, and improved discrimination. }\end{array}$ \\
\hline & ETAT & $\begin{array}{l}\text { Robertson } 2001[7] \\
\text { Malawi (Low Income) }\end{array}$ & $\begin{array}{l}n=2281 \text { Under } 5 \text { outpatient } \\
\text { clinic with referral for admission } \\
(85 \%<5 \text { y/o })\end{array}$ & $\begin{array}{l}\text { Overall: } 14.9 \% \text { P1: } \\
\text { 90.0\% P2: } 32.0 \% \\
\text { P3: } 4.5 \%\end{array}$ & $\begin{array}{l}\text { No follow-up data after admission. Only } \\
\text { patients under } 5 .\end{array}$ \\
\hline & PEWS & $\begin{array}{l}\text { Chaiyakulsil } 2015 \text { [16] } \\
\text { Thailand (Upper-Middle } \\
\text { income) }\end{array}$ & $\begin{array}{l}n=1136 \\
\text { ED at large tertiary hospital } \\
(100 \%<15 \mathrm{y} / 0)\end{array}$ & $\begin{array}{l}\text { Overall AUC: } 0.73 \\
\text { ICU: } 0.98 \\
\text { Ward } 0.71 \\
\text { PEWS > } 1 \text { for } \\
\text { admission } \\
\text { Sensitivity } 77 \% \\
\text { Specificity } 59 \%\end{array}$ & $\begin{array}{l}\text { Measured in area under ROC curve, for } \\
\text { sensitivity and specificity for admission by } \\
\text { PEWS category. }\end{array}$ \\
\hline & PATS & $\begin{array}{l}\text { Mullan } 2014 \text { [ ] } \\
\text { Botswana } \\
\text { (Upper-middle Income) }\end{array}$ & $\begin{array}{l}N=4466 \\
E D \text { at terteriary referral hospital } \\
(100 \%<13 \mathrm{y} / 0)\end{array}$ & $\begin{array}{l}\text { Overall: } 54.5 \% \\
\text { Red: } 86.7 \% \\
\text { Orange: } 66.1 \% \\
\text { Yellow: } 37.6 \% \\
\text { Green: } 20.6 \%\end{array}$ & $\begin{array}{l}\text { PATS performed at ED presentation. Large } \\
\text { study of both adult and pediatric patients } \\
\text { with separate analyses. }\end{array}$ \\
\hline
\end{tabular}

\section{Validity}

Six studies evaluated the validity of IMCI (Table 5) [19, 20, 24-27]. One study showed an increase in likelihood of admission according to urgency of triage level assigned [19]. Mazzi et al., reported that the sensitivity of individual clinical signs for predicting serious illness requiring hospital management was less than $35 \%$ for all signs except fever (65\%) [26].

\section{Discussion}

Overall, the quantity and quality of evidence to support the effectiveness of any single triage tool for pediatric patients in low resource settings is poor. This is driven by the limited number of studies available; the heterogeneous nature of these studies preventing formal meta- analysis; and the high proportion of studies conducted in urban centers in middle-income countries preventing extrapolation to low resource environments.

Our study identified a research gap in the quality and quantity of studies conducted in urban middle-income countries compared to rural environments in low-income countries, true low resource settings. Only one of the 16 studies were carried out in a low-income country [15] (Table 1). Although it is much more feasible to evaluate triage tools in high resourced district and tertiary care hospitals in middle-income countries (many of which resemble hospitals in high-income countries), these studies are difficult to extrapolate to rural low resource settings, where the need for these tools is greatest. In addition one may hypothesize that the triage tool may alter in their 
Table 4 Included Evaluations of Reliability of IMCI/IMNCI

\begin{tabular}{|c|c|c|c|c|c|}
\hline $\begin{array}{l}\text { Type of } \\
\text { Reliability } \\
\text { Evaluation }\end{array}$ & $\begin{array}{l}\text { Author[ref] Year } \\
\text { Country (Income } \\
\text { level) }\end{array}$ & $\begin{array}{l}\text { Comparison } \\
\text { Groups }\end{array}$ & $\begin{array}{l}\text { IMCI Variable } \\
\text { Being Assessed }\end{array}$ & $\begin{array}{l}\text { Volume Patient and Setting } \\
\text { Characteristics (Age } \\
\text { restrictions) }\end{array}$ & $\begin{array}{l}\text { Results (kappa (k), percent agreement (Pa), } \\
\text { Sensitivity (Sn), Specificity (Sp)) by triage level }\end{array}$ \\
\hline \multirow[t]{5}{*}{ Inter-Rater $^{a}$} & $\begin{array}{l}\text { Shewade [24] } \\
2013 \text { India (Lower- } \\
\text { Middle Income) }\end{array}$ & $\begin{array}{l}\text { 1. Health } \\
\text { Care Worker } \\
\text { 2. Study } \\
\text { Investigator }\end{array}$ & $\mathrm{IMCl}$ category & $\begin{array}{l}N=128 \\
\text { Community health center } \\
\text { (Omos-5 yrs) }\end{array}$ & $\begin{array}{l}\text { Overall } k=0.43 \\
<2 \text { mos } k=0.31 \\
>2 \text { mos } k=0.44\end{array}$ \\
\hline & $\begin{array}{l}\text { Battarachaya [21] } \\
2012 \text { India (Lower- } \\
\text { Middle Income) }\end{array}$ & $\begin{array}{l}\text { 1. Health } \\
\text { Care Worker } \\
\text { 2. Physician } \\
\text { (IMCI Red/ } \\
\text { Yellow) }\end{array}$ & $\begin{array}{l}\text { IMCI Red/Yellow } \\
\text { categorization }\end{array}$ & $\begin{array}{l}n=131 \text { Inpatient pediatric } \\
\text { hospital ( } 2 \text { mos-5 yrs) }\end{array}$ & $\begin{array}{l}\mathrm{Pa}=36 \% \\
\text { Simple } \mathrm{k}=0.16 \\
\text { Weighted } \mathrm{k}=0.29\end{array}$ \\
\hline & $\begin{array}{l}\text { Battarchaya [20] } \\
2013 \text { India (Lower- } \\
\text { Middle Income) }\end{array}$ & $\begin{array}{l}\text { 1. Health } \\
\text { Care Worker } \\
\text { 2. Physician } \\
\text { (Diagnosis) }\end{array}$ & $\begin{array}{l}\text { Diagnostic } \\
\text { category }\end{array}$ & $\begin{array}{l}n=117 \\
\text { Inpatient pediatric hospital } \\
(0-2 \mathrm{mos})\end{array}$ & $\begin{array}{l}\text { Serious Bacterial Infection } \\
\mathrm{k}=0.38 \\
\text { Local Bacterial Infection } \\
\mathrm{k}=0.20 \\
\text { Jaundice } \mathrm{k}=0.73 \\
\text { Dehydration } \mathrm{k}=0.19 \\
\text { Unable to Feed } \mathrm{k}=0.29\end{array}$ \\
\hline & $\begin{array}{l}\text { Battarchaya [19] } \\
2011 \text { India (Lower- } \\
\text { Middle Income) }\end{array}$ & $\begin{array}{l}\text { 1. Health } \\
\text { Care Worker } \\
\text { 2. Physician } \\
\text { (IMCI Red/ } \\
\text { Yellow) }\end{array}$ & $\begin{array}{l}\text { IMCI Red/Yellow } \\
\text { categorization }\end{array}$ & $\begin{array}{l}n=117 \\
\text { Inpatient pediatric hospital } \\
(0-2 \mathrm{mos})\end{array}$ & $\begin{array}{l}\mathrm{Pa}=56 \% \\
\text { Simple } \mathrm{k}=0.32 \\
\text { Weighted } \mathrm{k}=0.41\end{array}$ \\
\hline & $\begin{array}{l}\text { Gupta [23] } \\
2000 \text { India (Lower- } \\
\text { Middle Income) }\end{array}$ & $\begin{array}{l}\text { 1. Health } \\
\text { Care Worker } \\
\text { 2. Physician }\end{array}$ & $\begin{array}{l}\mathrm{IMCl} \\
\text { Categorization }\end{array}$ & $\begin{array}{l}n=129 \\
E D \text { and Outpatient clinics } \\
(0-2 \text { mos })\end{array}$ & $\mathrm{Pa}=60 \%$ \\
\hline \multirow[t]{4}{*}{$\begin{array}{l}\text { Gold } \\
\text { Standard } \\
\text { Comparison }^{b}\end{array}$} & $\begin{array}{l}\text { Mazzi [26] } \\
2010 \text { Bolivia (Lower- } \\
\text { Middle Income) }\end{array}$ & $\begin{array}{l}\text { 1. Triage } \\
\text { Nurse } \\
\text { 2. ED } \\
\text { Physician }\end{array}$ & $\begin{array}{l}\text { IMNCI Red within } \\
\text { Diagnostic } \\
\text { categories }\end{array}$ & $\begin{array}{l}n=1082 \\
2 \text { pediatric hospital ED } \\
\text { (Omos-2mos) }\end{array}$ & $\begin{array}{l}\text { Sn }<35 \% \text { for each sign } \\
\text { Sp }>85 \% \text { for all signs }\end{array}$ \\
\hline & $\begin{array}{l}\text { Battarachaya [20] } \\
2013 \text { India (Lower- } \\
\text { Middle Income) }\end{array}$ & $\begin{array}{l}\text { 1. ED Nurse } \\
\text { 2. Inpatient } \\
\text { Physician }^{\mathrm{a}}\end{array}$ & $\begin{array}{l}\text { IMNCI Red within } \\
\text { Diagnostic } \\
\text { categories }\end{array}$ & $\begin{array}{l}n=117 \\
\text { Inpatient pediatric hospital } \\
(0-2 \mathrm{mos})\end{array}$ & $\begin{array}{l}\text { Serious bacterial infection: } 89 \% \text { Sn, } \\
72 \% \text { Sp Local Bacterial Infection: 14\% } \\
\text { Sn, } 99 \% \text { Sp Jaundice: } 67 \% \text { Sn, } 99 \% \text { Sp } \\
\text { Dehydration: } 25 \% \text { Sn, 95\% Sp Poor feeding: } \\
44 \% \text { Sn, } 87 \% \text { Sp }\end{array}$ \\
\hline & $\begin{array}{l}\text { Mittal [27] } \\
2013 \text { India (Lower- } \\
\text { Middle Income) }\end{array}$ & $\begin{array}{l}\text { 1. Health } \\
\text { Care Worker } \\
\text { 2. ED } \\
\text { Physician }\end{array}$ & $\begin{array}{l}\text { IMCI Red } \\
\text { categorization }\end{array}$ & $\begin{array}{l}\mathrm{n}=1043 \\
\text { Outpatient and pediatric ED } \\
\text { in tertiary care center } \\
\text { (0mos- } 5 \mathrm{yr} \text { ) }\end{array}$ & $\begin{array}{l}38.7 \% \text { diagnostic mismatch in } 0-7 \mathrm{~d} \\
\text { age group } 24.3 \% \text { diagnostic mismatch } \\
\text { in } 7 \mathrm{~d}-2 \text { mos age group } 19.9 \% \text { mismatch } \\
\text { in } 2 \text { mos-5 yr }\end{array}$ \\
\hline & $\begin{array}{l}\text { Cao [22] } 2004 \\
\text { Vietnam (Upper- } \\
\text { Middle Income) }\end{array}$ & $\begin{array}{l}\text { 1. Health } \\
\text { Care Worker } \\
\text { 2. Physician }\end{array}$ & $\begin{array}{l}\text { IMCI Red } \\
\text { categorization }\end{array}$ & $\begin{array}{l}n=859 \\
\text { Inpatient pediatric hospital } \\
(2 \text { mos-5 yrs) }\end{array}$ & $\begin{array}{l}\text { Severe IIIness (IMCI Red) } \\
\text { Sn } 94.7 \% \\
\text { Sp } 96.1 \%\end{array}$ \\
\hline
\end{tabular}

${ }^{a}$ Inter-Rater reliability was measured as 2 individuals agreement without weight of importance. It is expressed in percent agreement or kappa statistics

${ }^{\mathrm{b}}$ Gold Standard Comparison expresses the ability of triage personnel (workers, nurses) to physicians. It is expressed in sensitivity and specificity

Table 5 Included evaluations of validity by real patient outcomes of $1 \mathrm{MCl} / \mathrm{MNCl}$

\begin{tabular}{llll}
\hline Outcome & Author [ref] Year Country (Income) & $\begin{array}{l}\text { Site Volume Patient and Setting } \\
\text { Characteristics (Age restrictions) }\end{array}$ & $\begin{array}{l}\text { Results (per triage level) } \\
{[p \text { value] }}\end{array}$ \\
\hline Admission & Battarachaya [19] & $\begin{array}{l}n=131 \text { Inpatient pediatric hospital ED } \\
(2 \mathrm{mos}-5 \text { yrs) }\end{array}$ & $\begin{array}{l}\text { Overall: 25\% } \\
\text { Red: } 82 \% \\
\text { Yellow: } 11 \% \\
\text { Green 5\% }\end{array}$ \\
& 2012 India (Lower-Middle Income) & & $95 \% \mathrm{Sn}$ \\
& & $n=419$ Outpatient pediatric clinic and ED & $87 \%$ Sp \\
Referral to ED & Kaur [24] & in tertiary care center (0-2mos) & $98 \% \mathrm{Sn}$ \\
& 2011 India (Lower-Middle Income) & $n=309$ Outpatient pediatric clinic and ED & \\
& Kundra [25] & in tertiary care center (2mos-5 yrs) & \\
\hline
\end{tabular}


function in low resource settings given differences in available treatments, training of providers, underlying healthcare system infrastructure and prevalent disease pathologies (i.e., high prevalence of HIV infection and malnutrition for example).

The tools included in this study varied in their construct. ETAT was an example of the use of clinical signs alone, without vital signs or any input of the presenting complaint. Tools based on clinical signs alone have appeal as they can be employed quickly in settings where measuring vital signs may be too time-consuming or impractical [10]. Conversely PEWS and TOPRS allow for a true objective provider assessment, which may be less likely to introduce bias and may be performed by providers with more basic training $[16,29]$. Then, tools such as the pSATS and PATS incorporate both clinical and physiologic data. The Pediatric South African Triage Scale (pSATS), incorporates the ETAT ABCD emergency signs as well as Triage Early Warning Score (TEWS) physiologic parameters to stratify patients. Twomey et al. studied the sensitivity of the pSATS tool, suggesting it is a more robust screening modality than either clinical discriminators or TEWS alone. The sensitivity (Sn) and negative predictive value (NPV) of pSATS was higher (91 and 93\%, respectively) when compared to clinical discriminators alone (Sn 57 and NPV 86\%) or TEWS alone (Sn 75.6 and NPV 89\%). Appropriately, children triaged to lowest category were correctly identified as non-urgent. Advocates of these mixed triage scores argue that the addition of vital signs significantly increases the sensitivity for identifying sick children and outweighs the additional time required [18].

Our original intent was to make comparisons between triage tools, and to meta-analyze the reliability and validity of tools in different settings. This was impossible due to the limited number of studies, and variability in study design. Reliability assessments varied in statistical analyses (from kappa statistics to percent agreement [15] to sensitivity/specificity of a binary outcome $[22,26]$ ), and methodology (comparison groups varied between studies, IMCI variable varied in reliability assessments) (Table 1, Table 3). This made formal meta-analysis impossible, and prevented a true global assessment of the reliability of any one triage tool.

A number of studies reported nursing triage to physician triage [17]. However the use of physicians as a gold standard in triage sensitivity does also give cause of concern. In most developed countries, a nursing professional is responsible for triage operations. Given the lack of healthcare provider resources, physicians in triage would be very unusual in LMICs. Therefore, it is unclear if the use of a physician as the gold standard for reliability measurements is appropriate in these studies. All reliability studies did however utilize real patients in their evaluations opposed to written scenarios, and may be the reason for poor reliability data that is reported. In adult patients the many reliability studies use pre fabricated written cases to assess reliability and thus report higher agreement [32, 33].

Validity studies also varied widely, where different methodologies prevented true meta analysis and study limitations hindered the quality of evidence. All validity studies had large sample sizes, ranging from 407 [10] to 4466 [31] participants. However, the methodology varied widely, with some triage applying to patients who were "admitted" to the ED (ie were expected to stay for a period of time) [29], while others were done at initial presentation. A major source of variability was the overall rates of mortality and admission at different locales. The studies measuring admission outcomes differed significantly in their overall admission rates from 9.4\% [34] to $55.4 \%$ [31]. Overall mortality at the study sites varied from $0.16 \%$ [31] to $16.3 \%$ [29].

Most studies rely on admission or mortality as a proxy for severity of illness. In low resource health care environments, there are numerous confounders that can impact outcomes including the training of providers, availability of medications and surgical interventions, availability of specialty/critical care, and the ability of patients to pay for treatment. In addition, the lack of follow-up data in any of these studies significantly hinders its effectiveness. For those patients not admitted, there is absolutely no data on mortality or re-presentation in any of the studies featured. Given resource and infrastructure constraints (census, patient records etc.), this is an understandable, but significant, limitation of the research in this field. Furthermore, this oversight is not merely restricted to studies in LMICs but also a limitation of several of the studies in the Farrokhnia review that focuses on high resource settings [5]. A systematic effort is required to overcome this limitation. Funders and investigators need to prioritize prospective evaluations with an emphasis on follow up of all patients that are triaged during the study period opposed to retrospective evaluations that only include admitted patients.

A single study by Molyneaux et al., does warrant special mention [6]. They demonstrated a near $50 \%$ reduction in under five-year-old inpatient mortality at a district hospital in Malawi after making improvements in triage, which included formal ETAT training. Although, in its true essence, this study does not evaluate the validity of a triage tool, the authors demonstrated that implementation of a triage system in their clinical environment significantly reduced overall child mortality. This study's validity cannot be appraised, as the authors do not provide specific information on the outcome of patients assigned to various categories. In addition, the pre/post study design is prone to multiple confounders given the many simultaneous changes to the triage system (new hires, better clinician 
oversight, new physical plant, etc.). These confounders make quantifying the effects of ETAT alone impossible [6].

In evaluating the evidence supporting IMCI, it is evident that, although numerous, there is insufficient evidence to validate the tool in varied low-resource environments, due to similar problems with heterogeneous study methods and numerous study limitations. Comparing IMCI to other pediatric triage tools, IMCI also addresses aspects of nutrition, immunization, and other important elements of disease prevention and health promotion. Accordingly, many of the IMCI studies considered in this review evaluate patients in an outpatient or clinic setting. Interestingly, the volume of studies evaluating IMCI far outnumber the literature on any of the triage tools, likely due to the 1994 WHO mandate to complete large multi-country evaluations on the training and implementation of IMCI worldwide [35].

Despite the relative plethora of studies validating IMCI, there are significant limitations in study design and locale. First, the IMCI studies are smaller in size, likely representing the relative patient census at the smaller centers in which the studies were conducted. In addition, these studies suffer from a lack of standard method for assessing IMCI reliability, and individual studies present varied methods of comparison, comparison groups, and IMCI variables to be compared (Table 3). This prevents a formal analysis of the overall IMCI reliability between raters.

Additionally, the kappa values for reliability are significantly lower than those reported in the triage tool studies (Table 3). One possible explanation is that IMCI is typically utilized by health care providers with less formal training, while the responsibility of triage in larger centers is often placed on professional nurses with formal schooling. In addition, numerous studies attempt to make validity conclusions based on IMCI performance compared to physician diagnosis as gold standard. In India, Kaur and others demonstrated that the IMNCI adaptation is a sensitive tool (95\%) for identifying neonates for referral $[25,36]$ It is concerning that so many studies opted to use non blinded physician opinion as the gold standard. Diagnostic agreement and decision to admit or refer are generally poor metrics for validation, given that they are inherently biased by the initial triage decision. Of the studies considered in this review, none followed patients to collect outcome data such as treatments required, length of stay, or mortality [20]. Taken cumulatively, all of these limitations prevent a formal analysis of the reliability and validity of IMCI, and thus limit the ability to recommend it for practitioners in low-resource settings.

\section{Limitations}

This review only includes studies that were published in the peer-reviewed literature available on databases searched. Second, other tools that may be used to prioritize the care for children in low-resource settings may not be referred to as "triage" tools. Recognizing this, authors performed a separate search for IMCI, but other similar approaches may exist that were not included. Studies not available in English language were not considered in this review. Relevant studies published without translation may have been excluded.

\section{Conclusions}

Overall, there is little in the literature studying the performance of triage tools in pediatric patients in low resource settings. The generalizability of these studies is also difficult given the preponderance of studies conducted in urban centers in middle-income countries opposed to true low resource settings. A large number of studies depend on the local physician assessment as a gold standard, which is highly variable and difficult to reproduce across studies. Thus it is difficult to support the use of a single tool based on this systematic review. Despite the methodological concerns evaluating IMCI studies, the ubiquitous use of IMCI as well as the availability of training and implementation though the WHO does support its continued use in outpatient clinic settings where it is currently implemented.

Overall studies evaluating triage tools in this vulnerable population are scarce and generally do not include follow up of lower acuity patients and critically important outcomes data. There is a need to develop and define robust validation methodology that can be prospectively utilized to evaluate triage tools in low resource settings.

\section{Additional files}

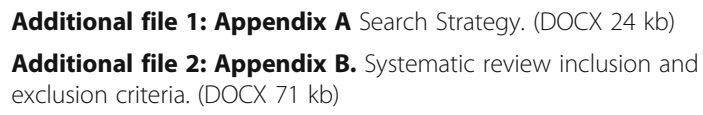

Additional file 3: Appendix C. Triage Tools Data Abstraction. (PDF $126 \mathrm{~kb}$ )

\begin{abstract}
Abbreviations
ED: Emergency department; ESI: Emergency severity index; ETAT: Emergency triage and treatment tool; ICU: Intensive care unit; IMCI: Integrated management of childhood Illness; LMIC: Low and middle income countries; MDG: Millennium development goal; NPV: Negative predictive value; PEWS: Pediatric early warning score; PSATS: Pediatric south african triage scale; Sn: Sensitivity; TEWS: Triage early warning score; TOPRS: Temperature, $\mathrm{O} 2$ saturation, pulse, respiratory rate, sensorium/seizures; WHO: World health organization
\end{abstract}

\section{Acknowledgements}

None.

Funding

There are no funding sources to declare.

Availability of data and material

All data generated or analyzed during this study are included in this published article in the Additional files. 


\section{Authors' contributions}

BH conceptualized and designed the study, participated in article selection designed the initial manuscript, and edited and approved the final manuscript as submitted. AJ conducted the literature search, participated in article selection, and drafted the initial manuscript. DK drafted the initia manuscript and developed the initial tables. SR, TA, MT participated in article selection and contributed to the writing of the manuscript. GDK and LW conceptualized the study and edited and approved the final manuscript.

\section{Competing interests}

The authors have no financial and non-financial competing interests to declare.

\section{Consent for publication}

Not Applicable.

\section{Ethics approval and consent to participate}

Not Applicable.

\section{Author details}

'Department of Emergency Medicine, Johns Hopkins School of Medicine, 1830 Monument St Suite 6-100, Baltimore, MD 21287, USA. ${ }^{2}$ University of Cape Town Division of Emergency Medicine, Private Bag X24, Bellville 7535 South Africa. ${ }^{3}$ Welch Medical Library, Johns Hopkins School of Medicine, Baltimore, USA.

Received: 14 May 2016 Accepted: 18 January 2017

Published online: 26 January 2017

\section{References}

1. You D, Hug L, Chen Y. Levels and trends in child mortality. UNICEF. New York: UNICEF; 2014.

2. Barasa EW, Ayieko P, Cleary S, English M. A multifaceted intervention to improve the quality of care of children in district hospitals in Kenya: a cost-effectiveness analysis. PLoS Med. 2012:9(6):e1001238.

3. Jones G, Steketee RW, Black RE, Bhutta ZA, Morris SS. Bellagio child survival study G. How many child deaths can we prevent this year? Lancet. 2003; 362(9377):65-71.

4. Riviello ED, Letchford S, Achieng L, Newton MW. Critical care in resource-poor settings: lessons learned and future directions. Crit Care Med. 2011;39(4):860-7.

5. Farrohknia N, Castren M, Ehrenberg A, et al. Emergency department triage scales and their components: a systematic review of the scientific evidence. Scand J Trauma Resusc Emerg Med. 2011;19:42.

6. Molyneux E, Ahmad S, Robertson A. Improved triage and emergency care for children reduces inpatient mortality in a resource-constrained setting. Bull World Health Organ. 2006:84:314-9.

7. Robertson MA, Molyneux EM. Triage in the developing world-can it be done? Arch Dis Child. 2001:85(3):208-13.

8. Rowe AK, de Savigny D, Lanata CF, Victora CG. How can we achieve and maintain high-quality performance of health workers in low-resource settings? Lancet. 2005;366(9490):1026-35.

9. Gottschalk SB, Wood D, DeVries S, Wallis LA, Bruijns S, Cape TG. The cape triage score: a new triage system South Africa. Proposal from the cape triage group. Emerg Med J. 2006;23(2):149-53.

10. Buys H, Muloiwa R, Westwood C, Richardson D, Cheema B, Westwood A. An adapted triage tool (ETAT) at red cross war memorial children's hospital medical emergency unit, cape town: an evaluation. S Afr Med J. 2013;103(3):161-5

11. Group TWB. Country and Lending Groups. Data 2014; https://datahelpdesk. worldbank.org/knowledgebase/articles/906519-world-bank-country-andlending-groups. Accessed 22 Dec 2014.

12. Dalwai MK, Tayler-Smith $\mathrm{K}$, Trelles M, et al. Implementation of a triage score system in an emergency room in timergara, Pakistan. Public Health Action. 2013:3:43-5

13. Rosedale K, Smith ZA, Davies H, Wood D. The effectiveness of the South African triage score (SATS) in a rural emergency department. S Afr Med J. 2011;101:537-40.

14. Bruijns SR, Wallis LA, Burch VC. A prospective evaluation of the cape triage score in the emergency department of an urban public hospital in South Africa. Emerg Med J. 2008;25:398-402.
15. Robertson MA, Molyneux EM. 1718902; description of cause of serious illness and outcome in patients identified using ETAT guidelines in urban Malawi. Arch Dis Child. 2001;85:214-7

16. Chaiyakulsil C, Pandee U. Validation of pediatric early warning score in pediatric emergency department. Pediatr Int. 2015;57(4):694-8.

17. Jafari-Rouhi AH, Sardashti S, Taghizadieh A, Soleimanpour H, Barzegar M. The emergency severity index, version 4, for pediatric triage: a reliability study in Tabriz Children's hospital, Tabriz, Iran. Int J Emerg Med. 2013;6(1):36

18. Twomey M, Cheema B, Buys $\mathrm{H}$, et al. Vital signs for children at triage: a multicentre validation of the revised South African triage scale (SATS) for children. S Afr Med J. 2013:103(5):304-8.

19. Bhattacharyya A, Chatterjee C, Chatterjee S, Dasgupta S. A comparative study of the management decisions by $\mathrm{IMNCI}$ algorithm and by pediatricians of a teaching hospital for the children between 2 months to 5 years. Indian J Community Med. 2012;37(3):174-9.

20. Bhattacharyya A, Mukherjee S, Chatterjee C, Dasgupta S. Assessment of validity and reliability of $\mathrm{IMNCl}$ algorithm in comparison to provisional diagnosis of senior pediatricians in a tertiary hospital of Kolkata. J Fam Med Prim Care. 2013;2(2):173-7.

21. Bhattacharyya A, Saha SK, Ghosh P, Chatterjee C, Dasgupta S. Study comparing the management decisions by $\mathrm{IMNCl}$ algorithm and pediatricians in a teaching hospital for the young infants between 0 to 2 months. Indian J Public Health. 2011;55(4):324-8.

22. Cao XT, Ngo TN, Kneen $R$, et al. Evaluation of an algorithm for integrated management of childhood illness in an area of Vietnam with dengue transmission. Trop Med Int Health. 2004;9(5):573-81.

23. Gupta R, Sachdev HP, Shah D. Evaluation of the WHO/UNICEF algorithm for integrated management of childhood illness between the ages of one week to two months. Indian Pediatr. 2000;37(4):383-90.

24. Kaur S, Singh V, Dutta AK, Chandra J. Validation of IMNCI algorithm for young infants (0-2 months) in India. Indian Pediatr. 2011:48(12):955-60.

25. Kundra S, Singh T, Chhatwal J. Utility of Indian adaptation of integrated management of childhood illness (IMCI) algorithm. Indian J Pediatr. 2008;75(8):781-5

26. Mazzi E, Bartos AE, Carlin J, Weber MW, Darmstadt GL. Bolivia clinical signs study G. Clinical signs predicting severe illness in young infants ( $<60$ days) in Bolivia. J Trop Pediatr. 2010:56(5):307-16.

27. Mittal K, Gupta V, Khanna P, Kaushik JS, Sharma A. Evaluation of integrated management of neonatal and childhood illness (IMNCI) algorithm for diagnosis and referral in under-five children. Indian J Pediatr. 2014;81(8):797-9.

28. Shewade HD, Aggarwal AK, Bharti B. Integrated management of neonatal and childhood illness (IMNCI): skill assessment of health and integrated child development scheme (ICDS) workers to classify sick under-five children. Indian J Pediatr. 2013:80(6):448-54.

29. Bains HS, Soni RK. A simple clinical score "TOPRS" to predict outcome in pediatric emergency department in a teaching hospital in India. Iran Pediatr. 2012;22:97-101.

30 Mullan PC. Reduced overtriage and undertriage with a new triage system in an urban accident and emergency department in Botswana: a cohort study. Personal communication with Bhakti Hansoti. Email: mullan20@gmail.com. 2016.

31 Mullan PC, Torrey SB, Chandra A, Caruso N, Kestler A. Reduced overtriage and undertriage with a new triage system in an urban accident and emergency department in Botswana: A cohort study. Emerg Med J. 2014; 31(5):356-60.

32 Twomey M, Mullan PC, Torrey SB, Wallis L, Kestler A. The princess marina hospital accident and emergency triage scale provides highly reliable triage acuity ratings. Emerg Med J. 2012;29:650-3.

33 Twomey M, Wallis LA, Thompson ML, Myers JE. The south African triage scale (adult version) provides valid acuity ratings when used by doctors and enrolled nursing assistants. Afr J Emerg Med. 2012;2:3-12.

34 Jafari-Rouhi A, Sardashti S, Taghizadieh A, Soleimanpour H, Barzegar M. The emergency severity index, version 4, for pediatric triage: a reliability study in Tabriz Children's hospital, Tabriz, Iran. Int J Emerg Med. 2013;6:36.

35 Bryce J, Victora CG, Habicht JP, Vaughan JP, Black RE. The multi-country evaluation of the integrated management of childhood illness strategy: lessons for the evaluation of public health interventions. Am J Public Health. 2004;94(3):406-15

36 Khanal B, Lewis O, Lewis M, Newbury J, Malla G. The Australasian triage scale applied in a tertiary care hospital in Nepal. Emerg Med Aust. 2005;17(1):88-9. 